\title{
Perspective on advances in resonance-region nuclear modeling and opportunities for future research
}

\author{
M.E. Dunn ${ }^{\mathrm{a}}$, N.M. Larson, H. Derrien, and L.C. Leal \\ Oak Ridge National Laboratory, P.O. Box 2008, Oak Ridge, TN 37831-6171, USA
}

\begin{abstract}
The advent of high-fidelity radiation-transport modeling capabilities, coupled with the need to analyze complex nuclear systems, has served to emphasize the importance of high-precision cross section data, including the associated covariance information. Due to the complex nature of resonance-region interactions, cross section data cannot be calculated directly from theory; rather, high-precision resonance-region cross section measurements must be made at facilities such as the Oak Ridge Electron Linear Accelerator (ORELA) at Oak Ridge National Laboratory (ORNL), Geel Electron Linear Accelerator (GELINA), Rensselaer Polytechnic Institute (RPI). To extract accurate cross section data from these measurements, detailed nuclear modeling of the measured data is performed to parameterize the cross section behavior in the resonance range. The objective of this paper is to highlight recent advances in resonance-region nuclear modeling with particular emphasis on the covariance analysis capabilities. Opportunities for future research are identified in an effort to stimulate further advances in the state of the art nuclear modeling capabilities.
\end{abstract}

\section{Introduction}

Basic science and nuclear applications continue to stimulate the need for improved nuclear data, including covariance information. For applied programs, novel nuclear applications have identified nuclear data needs that require new measurements and cross section evaluations. The advent of modern sensitivity/uncertainty $(\mathrm{S} / \mathrm{U})$ analysis tools, coupled with the lack of uncertainty data in the various international nuclear data files, has stimulated the need for cross section covariance data. Details concerning nuclear data needs for nuclear criticality safety and advanced reactor systems are described in two papers by McKnight [1] and Salvatores [2] at this conference.

The importance of improvements in nuclear data is underscored in another application: improvements in fission product (FP) nuclear data have been identified for supporting burnup credit (BUC) for spent nuclear fuel (SNF). BUC will allow a licensee to take credit for "burned" SNF in transportation, handling, and storage operations. In the US, the implementation of BUC could potentially save hundreds of millions of dollars in the cost of SNF transportation.

Over the years, significant efforts have been devoted to the nuclear data evaluation of major isotopes pertinent to reactor applications (i.e., uranium, plutonium, and other actinides). However, efforts to evaluate fission-product (FP) cross section data for other nuclides have been less rigorous than for the actinide data. Recent assessments for FP have highlighted the need for improved nuclear data (i.e., including covariance data) for ${ }^{103} \mathrm{Rh},{ }^{133} \mathrm{Cs},{ }^{153} \mathrm{Eu},{ }^{155} \mathrm{Eu},{ }^{143} \mathrm{Nd},{ }^{149} \mathrm{Sm},{ }^{151} \mathrm{Sm}$, and ${ }^{155} \mathrm{Gd}$. These FP isotopes alone can account for as much as $19 \%$ of reactivity worth in an SNF cask. Thus, nuclear data research will continue to have a significant impact on nuclear fuel cycle applications.

Regarding basic science needs, there continues to be an urgent demand for neutron nuclear astrophysics data in the

\footnotetext{
${ }^{a}$ Presenting author, e-mail: dunnme@ornl.gov
}

resonance region. Moreover, an ever increasing number of precision abundance determinations in meteorites and very old stars, recent changes and improvements in stellar models, and the realization of new ways in which neutron experiments yield vital nuclear astrophysics data all have contributed to the need for new experiments. New data have been used to test and improve models of astrophysical objects ranging from red giant stars to supernovae, and of the chemical evolution of the galaxy. However, many open questions remain. Much interesting and important work remains to be done, and there is significant overlap between the techniques and types of experiments needed for nuclear astrophysics and those needed for applied programs such as nuclear criticality safety, advanced reactor applications, and fission applications. As a result, both applied and basic science programs will benefit from improved nuclear modeling capabilities.

In the subsequent discussion, the status of resonanceregion nuclear modeling methods is presented along with recommendations for future development.

\section{The current status}

In recent years, resonance-region nuclear-modeling research has led to advances in analysis capabilities for providing resonance-parameter values and covariance data. As experimental techniques have improved, analysis techniques have also evolved and improved. Some of these techniques are discussed in later sections of this paper.

Significant effort has been devoted to quantifying uncertainty information in an effort to facilitate the calculation of realistic and accurate resonance-parameter covariance matrices (RPCMs). Both statistical and systematic uncertainties must be included in resonance-parameter uncertainty analyses. Statistical uncertainties arising from measurements contribute only to the diagonal of the data covariance matrix; off-diagonal contributions arise from multiple sources (e.g., systematic errors in cross section measurements, correlations due to the 
nuclear reaction formalism, etc.). Methods have been developed for efficient, accurate, and easy-to-use treatment of the full off-diagonal data covariance matrices during analyses in the resolved resonance region.

Recent nuclear modeling advances have facilitated more rigorous resonance-region analyses, which in turn made it necessary to improve the resonance-region formats for use in the Evaluated Nuclear Data File (ENDF/B) system. Two new formats have recently been approved: the format for resonance parameters permits the reporting of quite general R-matrix analyses, even those involving threshold reactions or charged-particle channels. The format for the resonanceparameter covariance matrix makes it possible to report even very large covariance matrices. More information on these formats is presented in ref. [3].

\section{Codes for data analysis in the resolved resonance region}

In the resolved resonance region, it is not possible to determine the structure of cross sections from first principles; there are no models that can confidently predict complex resonance behavior. Instead, evaluators must rely on phenomenological parameterization of measured data. For any given nuclide, there might be as many as several dozen measurements that are simultaneously analyzed in order to provide the best possible description of the underlying cross section.

Sophisticated computer codes are required for such evaluations. A number of codes have been developed for this purpose; each has its own particular strengths and weaknesses.

\section{SAMMY and REFIT}

The two codes in most common use for evaluation of neutron cross section data in the resolved resonance region are SAMMY [4] and REFIT [5]. Both use the Reich-Moore approximation to R-matrix theory for calculating the cross sections. Both codes have numerous options for adding corrections for experimental conditions, including Doppler and resolution broadening, multiple scattering corrections, and normalizations and backgrounds. In both codes, fitting of experiment to theory is accomplished via least-squares or generalized least-squares procedures, taking into account experimental uncertainties in the process.

There are major differences between the two codes. SAMMY uses analytic techniques whenever possible, including in the calculation of derivatives of the cross sections and in descriptions of the resolution functions. REFIT generally uses numerical techniques, which may permit more generality but also require more computation time and may be prone to numerical instabilities.

The REFIT code has limited capacity for treatment of uncertainties. The SAMMY code includes an extensive collection of options for including systematic experimental uncertainties [6]. Off-diagonal experimental data covariance matrices (DCMs) can be included explicitly or implicitly (by providing the various contributors to the DCMs). Alternatively, a measurement-related parameter can simply be flagged to inform the code that the parameter value is to be held fixed but its uncertainty is to be used as a contributor to the DCM. Parameters treated in this fashion are denoted "propagated uncertainty parameters" or PUPs. To first order, excluding any iterations for convergence, the least-squares formulae for the PUP treatment are mathematically equivalent to the leastsquares formulae for varying the parameter during the fitting process. Details are available in refs. [4] or [6].

EDA and RAC

Two codes, EDA [7] and RAC [8], were designed particularly for light-element evaluations. Both use the full R-matrix theory (as opposed to the Reich Moore or another approximation) for calculating the cross sections. Few measurement-related corrections are needed for these analyses; no Doppler broadening options and only limited resolution-broadening options are available in either code.

The EDA code includes normalization for each data set and incorporates the normalization uncertainties into the fitting process. The RAC code instead uses normalization uncertainties to derive the experimental data covariance matrices, which are then used in the fitting procedure. To first order, the two processes should be equivalent. However, because of differences in implementation plus the necessity of iteration, it is unlikely that the two codes would produce identical results even in very simple cases.

These two codes were used for evaluation of the lightelement standards for ENDF-VII, as part of a Coordinated Research Project (CRP) [9] under the auspices of the International Atomic Energy Agency (IAEA). Final results for cross sections and the associated covariance matrices were a composite of results produced by the two codes [10].

A third code, SAMMY [4], was used in the CRP to reconcile differences between the two treatments of uncertainties. SAMMY could not be used directly for the lightelement evaluations, primarily because it is not yet able to simultaneously analyze forward and inverse reactions. Instead, a series of comparisons tests were made using only data that all three codes could handle. Results of the comparisons indicate that (1) the EDA fitting procedure is similar (but not identical) to the SAMMY PUP procedure and (2) the RAC procedure is similar (but not identical) to the SAMMY explicit DCM method. The EDA code therefore has the more rigorous treatment. EDA also requires far less computation time, probably because the use of an explicit DCM is highly inefficient as demonstrated in ref. [6].

Other codes

Other R-matrix codes include ANARKI [11] at Saclay, a full R-matrix code, and RIDDLE [12], a full R-matrix code used for charged-particle analyses. The CONRAD code [13], currently under development at Cadarache, will use the ReichMoore approximation. There are surely other R-matrix codes of which the authors are unaware, and we apologize for their omission from this list.

\section{Treatment of uncertainties: recent advances and opportunities for future research}

The proper treatment of uncertainties is now, and has been for several years, a prime topic for research in the resolved resonance region. The resonance-region nuclear modeling 
process is the "bridge" that links the basic science nuclear data measurements with the "final" evaluated cross section data file that is ultimately used in radiation transport modeling tools. Therefore, the proper treatment of uncertainties in the experiments and in the subsequent analyses hinges on the ability of the nuclear modeling software and the evaluator to quantify and propagate the uncertainty information to the cross section covariance matrix (CSCM).

Beginning with experimental data, two different types of measurement-related uncertainties exist: (1) statistical and (2) systematic. Statistical uncertainties arise directly from the counting statistics of the measurement process and are described by the Poisson distribution. For a sufficiently large number of counts, the Poisson distribution is well approximated by a Gaussian (normal) distribution. Since each separate count has its own uncertainty, the contribution to the DCM from statistical uncertainties is strictly diagonal, and hence easily and accurately treated.

Systematic uncertainties, however, behave differently from statistical uncertainties. Systematic, or common, uncertainties arise from experimental conditions that affect the entire measurement rather than a single data point; uncertainties in normalization and backgrounds are prime examples. These uncertainties can be treated in a variety of different ways, thereby impacting the covariance analysis.

Taking the experimental data into account, the cross section data evaluation process produces the following results: (1) an R-matrix parameterization, including spin assignments, channel radii, and values for energies and widths of all resonances (including dummy resonances or other parameterization for external resonances), and (2) an associated resonance-parameter covariance matrix (RPCM) that defines the uncertainties in the parameter values and the correlations between the various parameters. The resonance parameters may then be used to reconstruct the "true" cross section without reference to any particular experiment, and the RPCM may be propagated through that calculation to give the CSCM.

As noted in the previous section, the proper-treatment-ofuncertainties topic was a major component of the CRP for light-element standards. One relatively direct result of that project was innovations in the treatment of DCMs within the SAMMY code; both the PUP capability and the implicit DCM methodology were developed because of ideas generated during the CRP. As a result, an evaluator now has the ability to propagate both statistical and systematic uncertainty information to the RPCM, thereby facilitating a more rigorous covariance analysis than was previously possible. Although significant strides have been made, various issues must still be addressed, and some pertinent issues are described in the following discussion.

Currently, a common perception is that CSCMs derived from R-matrix fits "always" have far "too small" uncertainties (square root of the diagonal elements of the CSCM). Various explanations have been offered for this phenomenon, none of them completely satisfying:

One of the authors (Larson) would argue (though not with great conviction) that at least three factors contribute to this phenomenon: (1) Not all sources of uncertainty have been included. Omitted sources include some experiment-related effects (e.g., cross correlations between two experiments performed at the same facility); biases built into the analysis codes (e.g., uncertainties associated with mistakes in the implementation of the resolution broadening calculation or with the crude approximations used for multiple scattering); and flaws in the analysis (e.g., missed resonances or wrong spin assignments). (2) Information is added, and uncertainty thereby decreased, by the use of a physical description such as R-matrix theory to describe the data. Therefore one should expect that evaluated cross sections have smaller uncertainties and different covariance matrices from those associated with measured data. (However, see the next paragraph for an important counter argument.) (3) The CSCM is not really too small. The misperception is based only on consideration of the uncertainties and not of the full off-diagonal covariance matrix; off-diagonal terms in the CSCM are generally very large relative to the diagonal elements.

Another of the authors (Derrien) would argue that it is not possible for the RPCM to convey all the uncertainty information. For example, in regions between resonances where the cross section is very small, the RPCM produces very small uncertainties for those cross sections. The small uncertainties accurately reflect the uncertainties that are propagated through the evaluation process, but they ignore one important consideration: The accuracy of any R-matrix analysis is based on the implicit assumption of complete accuracy for all of the quantum numbers and all the other "background" information. However, it is often possible to find two different sets of R-matrix parameters (involving different quantum number assignments) that give comparable good results for the resonance peaks but quite different results for the valleys between the peaks. The experimental data are insufficient to distinguish between the two sets. A methodology should therefore be designed to reflect that information in the CSCM. Derrien suggests that the addition of a systematic normalization and/or background uncertainty to the CSCM would provide a good representation of this otherwise non-quantifiable uncertainty.

Clearly more work is needed on the proper treatment of uncertainties, and an IAEA CRP would be a logical forum to further investigate the different sources of uncertainty and their impact on cross section covariance analyses.

Once the covariance analysis is complete, the communication of the RPCM is a separate but important issue. Formats exist within ENDF for reporting the RPCM (or the CSCM, in the case of light elements). For those few nuclides for which the RPCMs are unduly large, a new "compact" ENDF format is available; see refs. [3] or [14] for additional details. No format exists, however, for augmenting the RPCM as suggested above by Derrien.

Using available ENDF covariance formats, efforts are under way to provide reasonable estimates for RPCMs for important nuclides. For new evaluations, the RPCMs are determined directly via the fitting procedure (although small modifications may be made to accommodate some of the issues raised above). Because of time and manpower limitations, it is not possible to reevaluate all nuclides; instead, RPCMs must be retroactively estimated for existing evaluations. Techniques are available within SAMMY and other codes for doing so.

Recently, ORNL has developed RPCMs for ${ }^{233} \mathrm{U},{ }^{235} \mathrm{U}$, ${ }^{238} \mathrm{U}$, and ${ }^{239} \mathrm{Pu}$. Details concerning the resonance parameter covariance evaluation effort are published for ${ }^{233} \mathrm{U}$ [15], 
${ }^{238} \mathrm{U}$ [16] and ${ }^{239} \mathrm{Pu}$ [17], respectively. The authors plan to publish the resonance parameter covariance work for ${ }^{235} \mathrm{U}$ in the future. The ${ }^{239} \mathrm{Pu}$ work represents a reevaluation effort of the resonance parameters by Derrien, and the corresponding RPCM was prepared using the PUP option in SAMMY. Note that the paper concerning ${ }^{239} \mathrm{Pu}$ is published at this conference [17], and the reader should refer to this paper for a specific example that demonstrates the treatment of uncertainties in the evaluation effort.

\section{Other current research topics}

Development work for analysis of neutron cross sections in the resolved resonance region is a continuous process. As experiments improve, improvements are needed within the analysis codes to accurately describe subtle features that previously were not visible within the experimental data. Measurementrelated corrections that have always been included in the analysis codes (e.g., Doppler or resolution broadening) may require refinements to accurately reproduce the measured data.

For example, researchers at Geel [18] have studied the effect of the resolution function on the values of resonance parameters. They found that small but non-negligible differences occur in the resonance parameter values and recommended the addition of energy-dependent decay to the REFIT resolution function used for their study.

Evaluations try to make use of all existing data, and new methods of treatment may be needed for older data sets. A study at Geel [19] has suggested that dead-time, normalization, and background have sometimes been improperly treated during the data-reduction process. Often these can be retroactively corrected, either by modifying the data prior to the analysis or by including appropriate corrections within the analysis code.

One difficulty in using old data is the dearth of uncertainty information provided for those data. Recently, Borella et al. [20] proposed "a structure for EXFOR files resulting from TOF measurements which allows the storage of the covariance matrix with reduced storage space". "TOF" is "time-of-flight" and EXFOR is the name for the experimental nuclear reaction data base at Brookhaven National Laboratory.) Adoption and use of such a format for EXFOR data files would prevent future problems of this nature.

\section{Summary and conclusions}

Although this paper is not an exhaustive discussion of resonance-region nuclear modeling methods, the objective is to qualitatively assess the current status of uncertainty analysis capabilities and highlight opportunities for further research and development. Currently, new nuclear data experiments are in progress or are being planned to address resonanceregion nuclear data needs for advanced nuclear fuel cycle applications and astrophysics research. Clearly, there is a basic science and nuclear fuel cycle application basis for improved nuclear data with cross section covariance information. Moreover, advanced radiation transport analysis tools (i.e., including $\mathrm{S} / \mathrm{U}$ methods) coupled with modern computing systems continue to place greater demands on the underlying nuclear data. As a result, state-of-the-art nuclear modeling capabilities are absolutely essential for analyzing the measured data to prepare an accurate, high-fidelity R-matrix parameterization of all resonances along with the corresponding resonance parameter covariance matrix. As noted in the paper, significant progress has been made in recent years to improve resonanceregion covariance analysis capabilities; however, significant challenges remain and provide opportunities for future nuclear modeling development.

This work was sponsored by the US Department of Energy Nuclear Criticality Safety Program (NCSP), under contract DEAC0500OR22725 with UT-Battelle, LLC.

\section{References}

1. R. McKnight, M.E. Dunn, R. Little, J. Felty, J. McKamy, Nuclear Data Needs within the US Nuclear Criticality Safety Program (these proceedings).

2. M. Salvatores, G. Aliberti, G. Palmiotti, D. Rochman, P. Oblozinsky, M. Hermann, P. Talou, T. Kawano, L. Leal, A. Koning, I. Kodeli, Nuclear Data Needs for Advanced Reactor Systems. A NEA Nuclear Science Committee Initiative (these proceedings).

3. N.M. Larson, L.C. Leal, R.O. Sayer, H. Derrien, D. Wiarda, G. Arbanas, Current Status of the R-Matrix Code SAMMY, with Emphasis on the Relationship to ENDF Formats (these proceedings).

4. N.M. Larson, Updated Users' Guide for SAMMY: Multilevel R-Matrix Fits to Neutron Data Using Bayes' Equations, ENDF364/R1 and ORNL/TM-9179/R7, Oak Ridge National Laboratory, Oak Ridge, TN (2006).

5. M.C. Moxon, REFIT2: A Least Squares Fitting Program for Resonance Analysis of Neutron Transmission and Capture Data, NEA-0914/02, July 1989.

6. N.M. Larson, Use of Covariance Matrices in SAMMY, Workshop on Nuclear Data Evaluation for Reactor Applications (WONDER 2006), Château de Cadarache, October 9-11, 2006.

7. G.M. Hale, D.C. Dodder, K. Witte, Los Alamos National Laboratory, Los Alamos, New Mexico, USA (private communication, 1975).

8. Z.P. Chen, Reduced R-Matrix Analysis for ${ }^{17} \mathrm{O}$ system, At. Energy Sci. Technol. 29, 366 (1995).

9. A. Carlson et al., An International Neutron Cross Section Standards Evaluation (these proceedings).

10. E. Gai, V. Pronyaev, Uncertainty Justification of Neutron Cross Section Standards (these proceedings).

11. E. Berthoumieux, Saclay (private communication to N.M. Larson, 2006).

12. T.N. Massey, J.F. Guillemette, S.M. Grimes, J.E. O'Donnell, $R$-Matrix Analysis of the ${ }^{14} \mathrm{C}$ System, ND2004 (International Conference on Nuclear Data for Science and Technology, Sept. 26-Oct. 1, 2004), page $450 \mathrm{ff}$.

13. C. De Saint Jean, Status of CONRAD, a Nuclear Reaction Analysis Tool (these proceedings).

14. N.M. Larson, SAMMY User Guidance for ENDF Formats, ENDF367 and ORNL/TM2007/23, Oak Ridge National Laboratory, Oak Ridge, TN (March 2007). 
15. L.C. Leal, G. Arbanas, H. Derrien, N.M. Larson, B. Rearden, Covariance Data for ${ }^{233} U$ in the Resolved Resonance Region for Criticality Safety Applications, Mathematics and Computation, Supercomputing, Reactor Physics and Nuclear and Biological Applications, Palais des Papes, Avignon (M\&C 2005), France, September 12-15, 2005

16. L. Leal, H. Derrien, N. Larson, G. Arbanas, R.O. Sayer, ORNL Methodology for Covariance Generation for Sensitivity/Uncertainty Analysis, to be presented at the International Conference on Nuclear Criticality Safety, May-June 2007, St. Petersburg, Russia.

17. H. Derrien, L.C. Leal, N.M. Larson, ${ }^{239} \mathrm{Pu}$ Neutron Resonance Parameters Revisited and Covariance Matrix in the Neutron Energy Range Thermal to $2.5 \mathrm{keV}$ (these proceedings).
18. S. Kopecky, D. Ene, P. Schillebeeckx, The impact of the resolution function on the determination of resonance parameter, Workshop on Nuclear Data Evaluation for Reactor Applications (WONDER 2006), Château de Cadarache, October 9-11, 2006.

19. M. Moxon et al., Evaluation Using REFIT, Workshop on Nuclear Data Evaluation for Reactor Applications (WONDER 2006), Château de Cadarache, October 9-11, 2006.

20. A. Borella, C. Bastian, F. Gunsing, J. Heyse, G. Noguere, P. Schillebeeckx, AGS, A Code to Propagate Uncertainty of Cross Section Measurement Data, Workshop on Nuclear Data Evaluation for Reactor Applications (WONDER 2006), Château de Cadarache, October 9-11, 2006. 